\title{
Wearable Biopotential Measurement Using the TI ADS1198 Analog Front-End and Textile Electrodes
}

\author{
Signal conditioning and signal quality assessment
}

\author{
T. Pereira, H. Carvalho, A.P.Catarino, M.J.Dias \\ School of Engineering \\ University of Minho \\ Guimarães, Portugal \\ helder@det.uminho.pt
}

\begin{abstract}
The development of mobile systems for monitoring bioelectric signals outside a hospital environment involves many challenges that do not arise when it is in a controlled environment, like a hospital. The dimensions of these systems are an important factor to consider in order to facilitate their use without interfering with the daily activities of individuals.

The purpose of this work is the implementation of a singlesupply battery-powered, low power ECG/EMG signal monitoring system based on the ADS1198 Analog Front-End from Texas Instruments. The system was designed to acquire ECG signals from three electrodes using the integrated Right-Leg-Drive (RLD) circuit from the ADS1198.

The developed analog front-end was connected for testing purposes through the SPI interface to a NI-USB 8451 board and signals were acquired using LabVIEW. The circuit was tested in several situations and proved to provide high quality signals using textile integrated electrodes and conventional disposable gel electrodes.
\end{abstract}

Keywords-ECG, EMG, biopotential measurement, e-textiles

\section{INTRODUCTION}

The main objective of this work is the evaluation of the requirements and performance of the $\operatorname{ADS} 119 x / 129 x$ as a multichannel three-lead Electrocardiography / Electromyography (ECG/EMG) measurement device for etextile applications. In particular, the acquisition of ECG and multiple EMG signals using wearable electrodes is aimed for. The ADS119x/129x family provides up to 8 channels of threelead ECG/EMG acquisition, being thus an interesting option for the application in hand. However, this chip has been introduced to the market very recently and few works have reported on its application. In this work, an evaluation of its performance and requirements in terms of external circuitry for a battery-powered application is examined to assess its potential as a conditioning chip for e-textile applications such as the Bioswim instrumented swimsuit [1][2] or other EMG/ECG monitoring applications[3][4].

The authors wish to thank funding by FEDER- "Programa Operacional Factores de Competitividade - COMPETE", FCT - Fundação para a Ciência e a Tecnologia, projects PEstC/CTM/UI0264/2011 and PTDC/EEA-ELC/70803/2006, and Instituto de Telecomunicações.

\author{
O.Postolache ${ }^{1}$, P.S.Girão ${ }^{2}$ \\ Instituto de Telecomunicações/ISCTE ${ }^{1}$, DEEC-IST ${ }^{2}$ \\ $\mathrm{IUL}^{1}, \mathrm{UTL}^{2}$ \\ Lisbon, Portugal \\ opostolache@1x.it.pt
}

\section{STATE OF THE ART}

\section{A. Biopotential signal conditioning}

The measurement of biopotential signals presents several challenges because of the presence of electrical noise everywhere, especially produced by power systems. Several authors proposed models describing the sources of interference due to the power system.[5][6][7][8].

Fig. 1 represents a simplified model of the electrical field coupling between the power lines and the developed measurement system. $\mathrm{C}_{3}$ represents the capacitive coupling between power lines and the measurement system. In grounded systems, the current through $\mathrm{C}_{3}$ flows to ground and does not cause interference. The proposed system is battery operated, which means that it is isolated from the earth ground. This leads to an interference current, $\mathrm{Id}_{3}$, which flows to earth ground through $\mathrm{C}_{4}$ and $\mathrm{C}_{5}, \mathrm{C}_{1}$ and $\mathrm{C}_{2}$ represent the capacitance between the power line and the lead wires. The currents $\mathrm{I}_{\mathrm{d} 1}$ and $\mathrm{I}_{\mathrm{d} 2}$, produced by capacitive coupling, do not flow into the electrocardiograph because of its high input impedance. Instead, they flow to the body through $Z_{1}$ and $Z_{2}$, respectively. The path to the earth ground for these currents is closed with capacitance $\mathrm{C}_{4}$ and $\mathrm{C}_{5}, \mathrm{Z}_{1}, \mathrm{Z}_{2}$ and $\mathrm{Z}_{3}$ are the skin-electrode impedances.

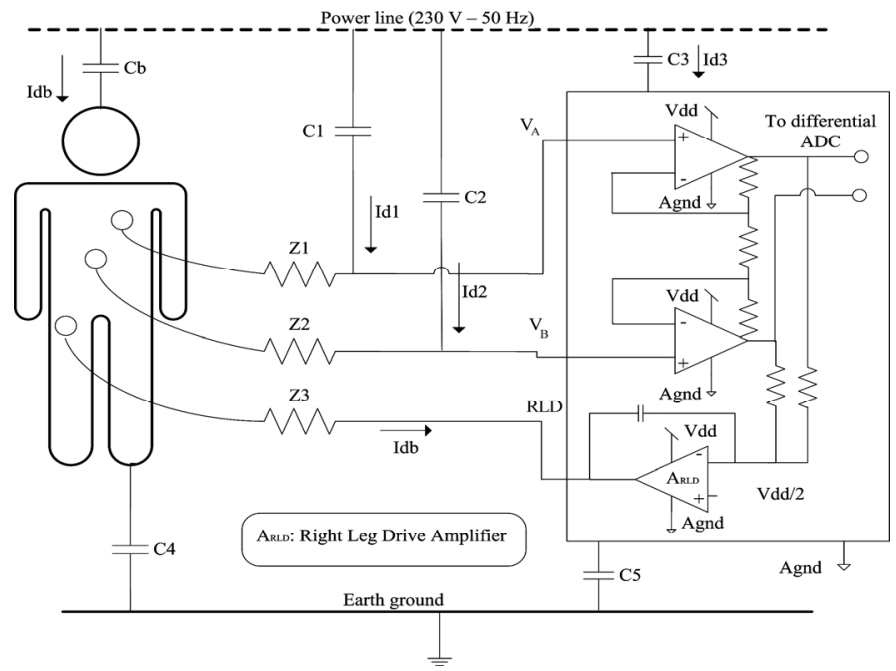

Fig. 1. Electrical coupling between power lines and measurement system 
According to [9] the differential voltage produced by this coupling can be calculated by equation (1):

$$
\mathrm{V}_{\mathrm{A}}-\mathrm{V}_{\mathrm{B}}=\mathrm{I}_{\mathrm{d} 1} \mathrm{Z}_{1}-\mathrm{I}_{\mathrm{d} 2} \mathrm{Z}_{2}
$$

To minimize this electrical interference, the impedances must be reduced and matched as closely as possible by reducing the skin-electrode impedance. The capacitive coupling can be reduced by using shielded signal connections [9].

When the electrical field coupling is established directly between the power lines and the body, a common mode voltage, $\mathrm{V}_{\mathrm{cm}}$ appears throughout the body due to current $\mathrm{I}_{\mathrm{db}}$. This current flows to ground through capacitance $\mathrm{C}_{4}$ and through $\mathrm{Z}_{3}$ via capacitance $\mathrm{C}_{5}$, [9][14].

For this reason it is important to select an amplifier with a high value of CMRR in order to improve the rejection of the common mode voltage. The influence of $\mathrm{V}_{\mathrm{cm}}$ can be also decreased by balancing the skin/electrode impedances $Z_{1}$ and $\mathrm{Z}_{2}$ as shown by equation (3).

$$
V_{A}-V_{B}=V_{c m} \frac{Z_{1}}{Z_{\text {in }}+Z_{1}}-\frac{Z_{2}}{Z_{\text {in }}+Z_{2}}
$$

and being $\mathrm{Z}_{1}$ and $\mathrm{Z}_{2}<<\mathrm{Z}_{\mathrm{in}}$ :

$$
V_{A}-V_{B}=V_{c m}\left(\frac{Z_{2}-Z_{1}}{Z_{\text {in }}}\right)
$$

Equation (3) demonstrates the importance of the balance between skin/electrode impedances $Z_{1}$ and $Z_{2}$. A further important factor is the input impedance, $Z_{\text {in }}$, of the amplifiers.

Another way to reduce the influence of common-mode noise is by using a right-leg drive (RLD) circuit. An RLD circuit averages the voltage of the differential electrode pair to sense the common mode voltage. This voltage is then amplified, inverted and fed back to the body via the third electrode (RLD electrode)[10].

A further source of noise is the induction of currents into the cables by magnetic coupling. This can be reduced by using twisted wires.

\section{B. Analog Front End (AFE) Integrated Circuits (ICs)}

Few AFE ICs specifically designed for biopotential measurement are commercially available. The ADS119x/129x family from Texas Instruments and RHA1016 from Intan Technologies, are two examples of these specialized ICs.

The application of AFE ICs in portable biopotential measurement devices makes it possible to reduce size, complexity and power consumption of the system. In [11] the authors used the RHA1016 in a wearable ECG recording system. The RHA1016 provides 16 differential amplifiers with a fixed gain of 200. This IC only requires two external resistors to configure the built-in programmable low-pass filter. The RHA1016 has an analog multiplexed differential output that must be connected to an external ADC.

The ADS119x/129x AFEs ICs family includes up to 8 differential channels with integrated ADCs for each channel, thus reducing the external components needed to configure a biopotential measurement system. In [12] the ADS1298 was integrated into a portable system for ECG monitoring.

\section{SYSTEM DEVELOPMENT}

As mentioned before, the ECG system developed in this work is based on ADS1198 from Texas Instruments.

The ADS119x is a family of multichannel, simultaneous sampling, 16-bit, delta-sigma (DS) analogue-to-digital converters (ADCs) with a built-in programmable gain amplifier (PGA), internal reference, and an onboard oscillator. In this work, the ADS1198 with 8 analogue differential channels has been used. The ADS1198 has a flexible input multiplexer that can be independently connected providing any configuration for the derivation of the right leg drive (RLD) output signal. The IC includes a SPI compatible serial interface for communication.

Additionally, the ADS1198 includes internal circuits allowing lead-off detection and pace detection. Three integrated amplifiers generate the Wilson Center Terminal (WCT) and the Goldberger terminals (GCT) required for a standard 12-lead ECG, a feature that has not been used in this work.

The ADS1198 has a maximum programmable gain of 12 . This gain is quite low considering the typical maximum amplitude of ECG and EMG signals, which is in the order of a few millivolts. To take advantage of the full dynamic range of the chip's ADC and provide more gain adjustment range, a preamplifier stage was developed.

\section{A. General system setup}

The system is composed of a differential pre-amplifier with AC-coupling and a differential low-pass filter connected to one of the channels of the ADS1198. The ADS1198 is connected via SPI to a NI USB-8451 board from National Instruments that provides an interface between SPI devices and a PC. Software in LabVIEW allows configuring the ADS1198, acquiring and storing data. Fig. 2 shows the general set-up of the system.

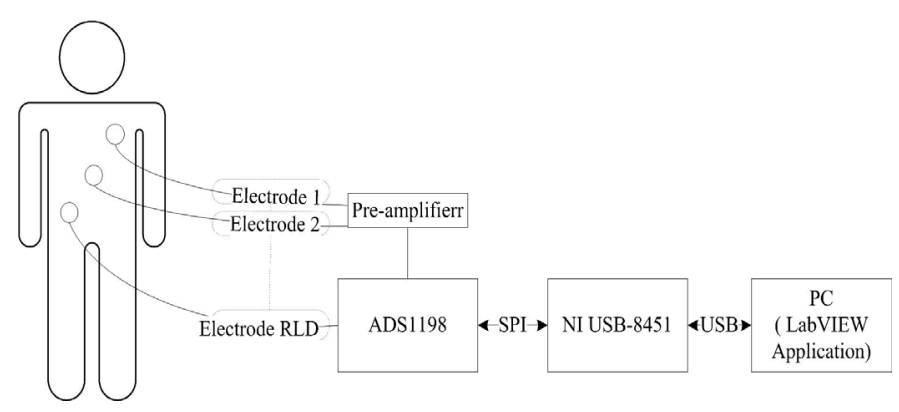

Fig. 2. General set-up 


\section{B. Pre-amplifier development}

The pre-amplifier was designed according to the work proposed in [13] to connect to one of the channels of the ADS1198 providing additional gain and AC coupling. The preamplifier can be dived in 4 different stages (Fig. 3):

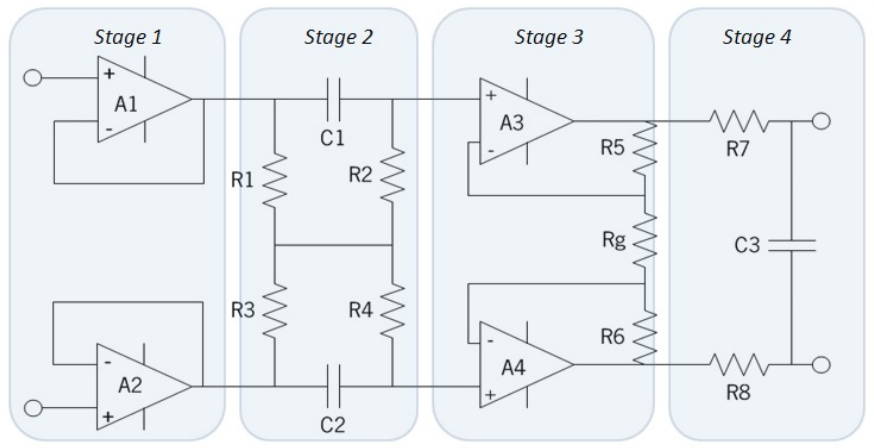

Fig. 3. Pre-amplifier

- Stage 1: This stage is a unitary buffer, providing a high impedance interface for the ECG electrodes.

- Stage 2: In this stage a differential ac-coupling network not requiring any resistors connected to ground, was implemented, thus optimizing CMRR. This network was proposed in [13]. It implements a first order highpass filter, designed for a cut-off frequency of $\mathrm{f}_{\mathrm{hp}}=0.028$ $\mathrm{Hz}$.

- Stage 3: A differential input - differential output amplifier with fixed gain $\mathrm{A}=28$, for differential signals.

- Stage 4: A first order differential low pass filter for avoiding aliasing effects on the acquired signal, designed for a cut-off frequency of $\mathrm{f}_{\mathrm{lp}}=523 \mathrm{~Hz}$.

The pre-amplifier stage was implemented with only one IC packing four operational amplifiers. The LMP7704 has been selected for its good features for biopotential signal conditioning. The LMP7704 are very low noise amplifiers with a typical input offset voltage of $\pm 56 \mu \mathrm{V}$. They feature a high CMRR of $130 \mathrm{~dB}$ and very low input bias current, $\pm 0.2 \mathrm{pA}$, and input offset current, 40fA.

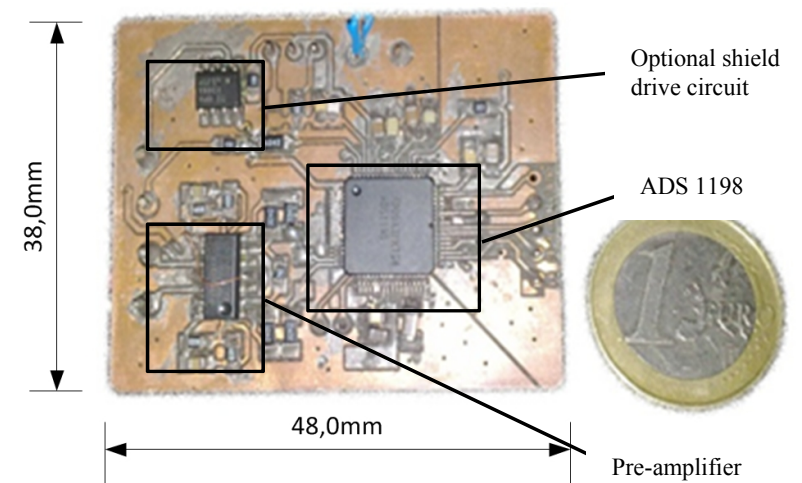

Fig. 4. PCB dimensions and components. (Optional shield drive circuit was not used in this work)

\section{Right-Leg drive circuit}

The ADS1198 provides an internal right-leg drive circuit. This signal is derived from any combination of the input signals. In this specific case, the RLD signal is derived from the two inputs of channel 1. For debugging purposes, the RLD output signal is externally routed to the RLD input of the ADS1198 and was acquired by the ADS1198 simultaneously with the ECG signal. The final PCB can be observed in Fig. 4.

\section{Electrodes}

Besides the standard disposable adhesive gel-electrodes (Spes Medica DENIS $15 \times 20 \mathrm{~mm}$ ), a shirt integrating textile electrodes knitted directly into the fabric was tested (Fig. 5.) The middle and lower right electrodes are the sensing electrodes whilst the lower left electrode is the RLD electrode.

For better signal quality, the shirt has been constructed so that it has a tight fit. In order to obtain a good contact and stabilization of the electrode, the shirt was produced with polyamide and bare elastane. These two raw materials combined with the knitted structure allowed localized compression effects and thus reduce some artifacts that are present with body movement.

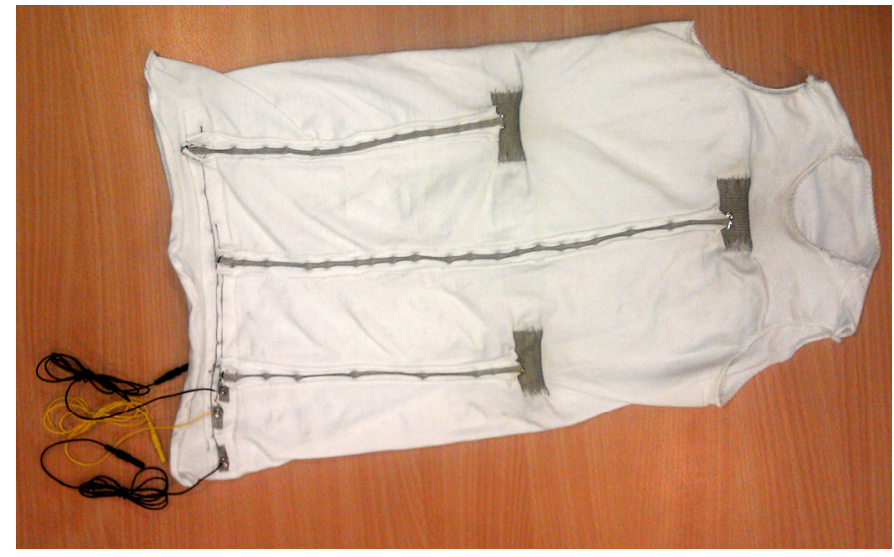

Fig. 5. Shirt with integrated textile electrodes

The electrodes are knitted by a MERZ model MBS seamless knitting machine which allows control of the yarn in terms of going in or out the knitting zone. Being a jacquard machine, it is possible to obtain complex structures with localised variations. This feature has been used to produce a particularly voluminous structure at the area used as electrode. In this way, the electrode area protrudes from the rest of the fabric, improving contact between the skin and the electrode, which is known to be a problem when using compression garments to stabilize the electrode's position.

The electrode areas are knitted with Elitex, which is a textured multifilament polyamide yarn with a thin silver coating (less than $10 \mathrm{~nm}$ ). They present very low values of electrical resistance (in the order $30 \Omega / \mathrm{m}$ ). On the other hand, they present stable behaviour in terms of impedance even when subjected to stress. 
The same knitting technology allows the integration of electrical connection lines in the textile substrate. Due to the process that involves the insertion of the yarn into the knitting zone and the individual movement of the knitting elements, namely the needles, horizontal connections are easier to produce than vertical connections (see Fig. 5). Vertical connections are possible, but due to the specificities of this particular machine, imply a much higher consumption of the expensive conductive yarn used. Instead, two possibilities exist. The first one is to create conduits with the normal, nonconductive yarns, through which conventional electrical conductors can be brought in. In this work, horizontal lines were created in a separate fabric. These were then cut and sewn onto the shirt in a vertical direction. To connect the electrodes, sewn vertical lines and knitted horizontal lines, and to ultimately connect the required signal cables, normal and magnetic snap fasteners are used (see Fig. 6)

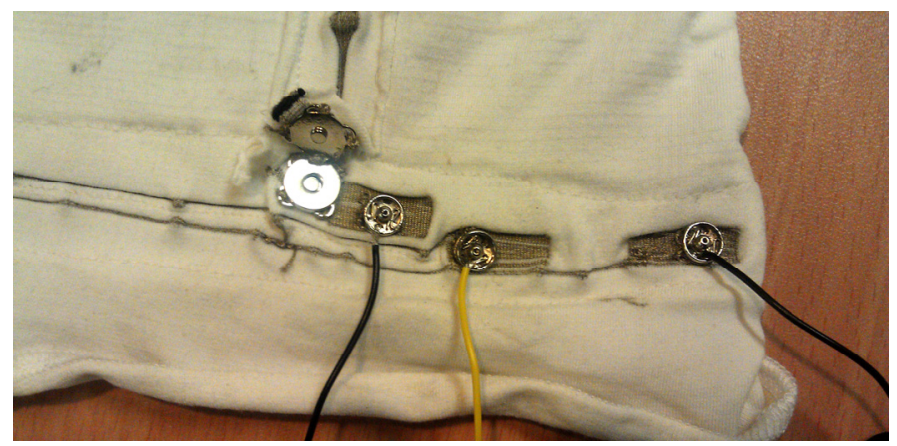

Fig. 6. Connection details

\section{E. LabVIEW application}

A simple LabVIEW application was developed to program and communicate with the ADS1198 with the desired operational configuration, and to perform signal acquisition, display and storage (Figures 7 and 8). Programming and data transfer to the PC are done through the SPI bus, accessed by the NI-8451 board. For the specific application in this work, the ADS1198 was set up as follows:

- Internal reference configured to $2.4 \mathrm{~V}$

- Data rate: $1000 \mathrm{~S} / \mathrm{s}$

- RLD signal generated from the 2 inputs of channel 1

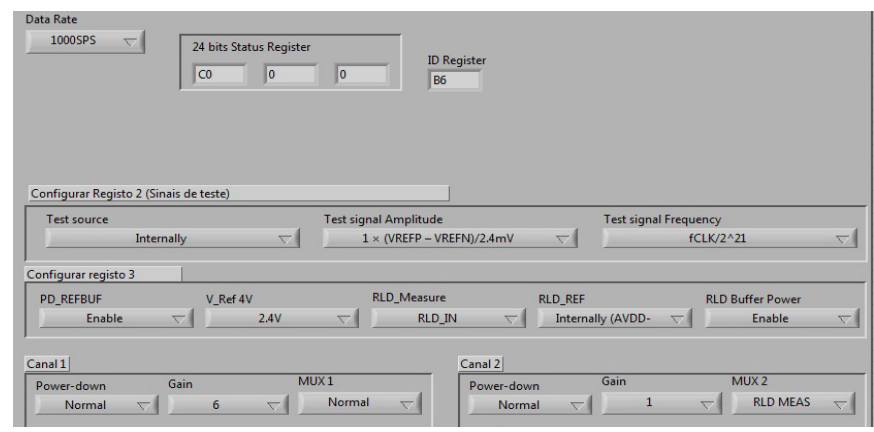

Fig. 7. ADS configuration panel in LabVIEW

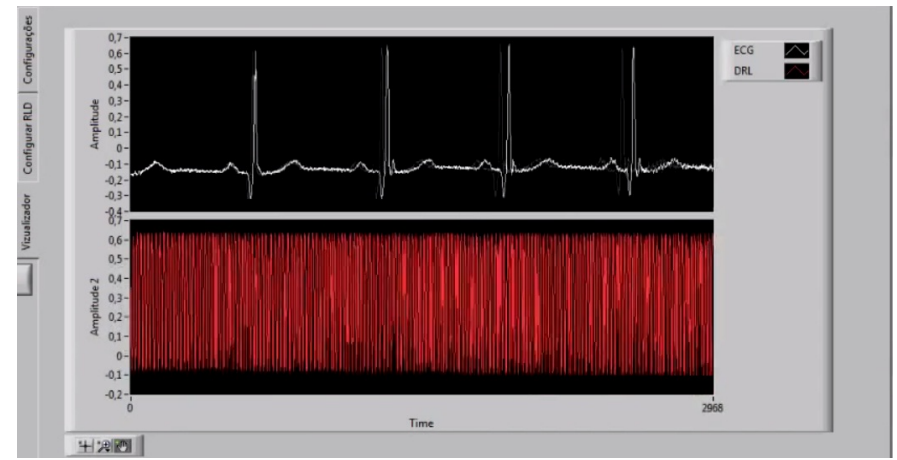

Fig. 8. Screenshot of the software - ECG and RLD output signal

The NI-USB 8451 does not support data streaming mode, thus each sample is transferred in an explicit read cycle. A data frame includes one sample of each channel configured for acquisition (channel 1 and RLD in, in this specific case), and a header with the chips internal state bits. Between two consecutives read cycles, the ADS1198 imposes a delay of about $0.9 \mathrm{~ms}$ before the next data frame is transferred. This results in a maximum usable sample rate of $1000 \mathrm{~S} / \mathrm{s}$ when using the NI-USB 8451 board.

\section{RESUlts}

\section{A. CMRR and noise analysis}

The CMRR and Input Referred Noise peak-to-peak (IRN $\mathrm{Ip}_{\mathrm{p}}$ ) of the system composed of the pre-amplifier and the ADS1198 were measured for a gain of $168(A=28 \cdot 6$, half of the maximum gain). 9:

The CMRR was measured using the circuit depicted in Fig.

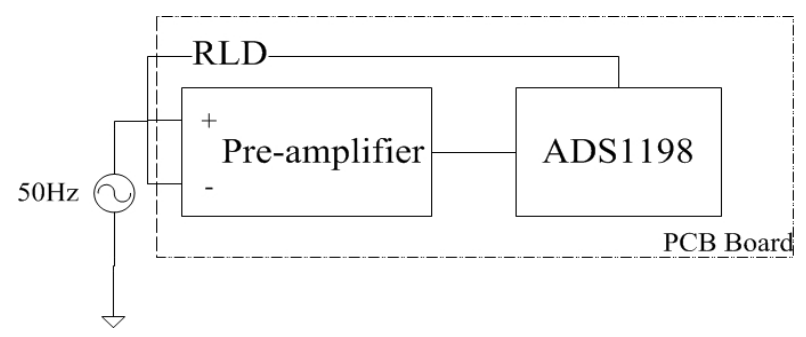

Fig. 9. Measurement of CMRR

The final measured value of CMRR was $93.8 \mathrm{~dB}$ with RLD and $73.4 \mathrm{~dB}$ without RLD.

The IRN $N_{p p}$ was measured with the configuration shown in Fig. 10:

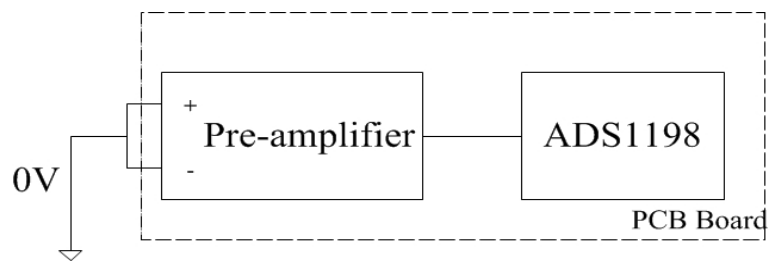

Fig. 10. Measurement of noise 
The measured input referred noise level was about $0.83 \mu \mathrm{V}$, which corresponding to about $2 \mathrm{LSB}$.

\section{B. Practical test: Signal acquisition using conventional electrodes, dry and wet textile electrodes}

Figures 11 to 13 show some of the experiments acquiring the ECG signal acquired with conventional, wet textile and dry textile electrodes. The signals are presented as acquired, not being subjected to any post-processing. The measurement system was electrically supplied by the laptop's USB port operating only on battery, disconnected from the mains power supply.

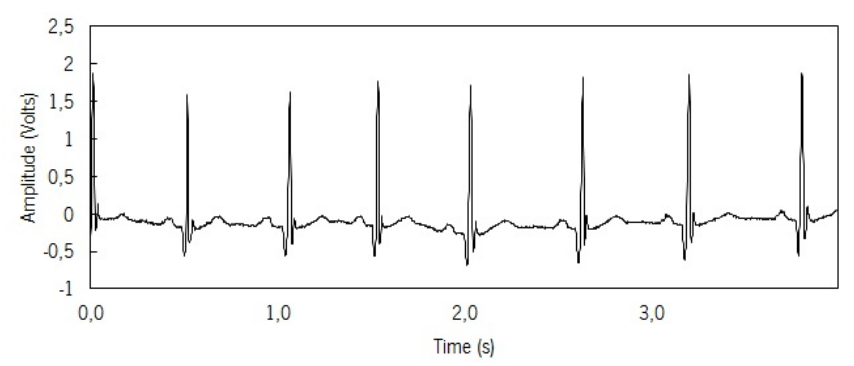

Fig. 11. ECG signal acquired with conventional gel-electrode

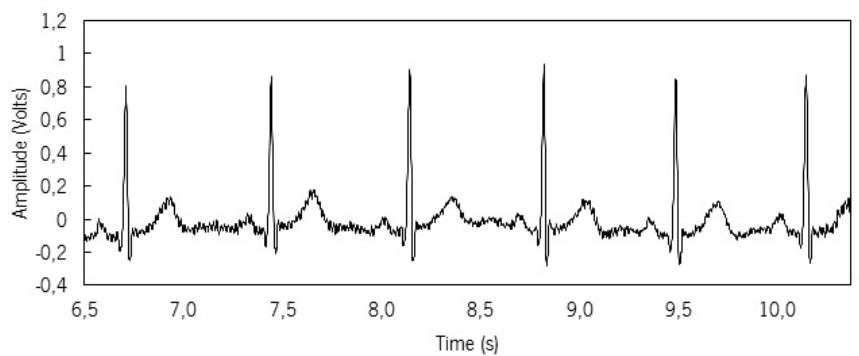

Fig. 12. ECG signal acquired with wet textile electrode

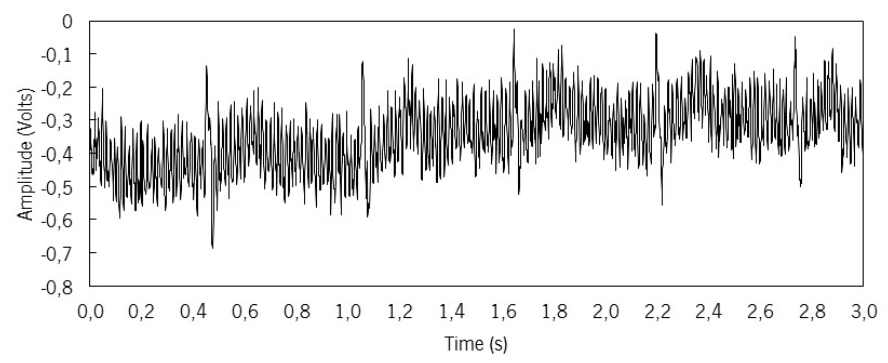

Fig. 13. ECG signal acquired with dry textile electrode

\section{Motion artifacts, $50 \mathrm{~Hz}$ noise and electrode disconnection}

To evaluate the effect of motion artifacts in the ECG signal, a test was made acquiring the ECG signal from the shirt with wet integrated textile electrodes, performing slow rotational movements with the body. Fig. 14 shows the resulting signal.

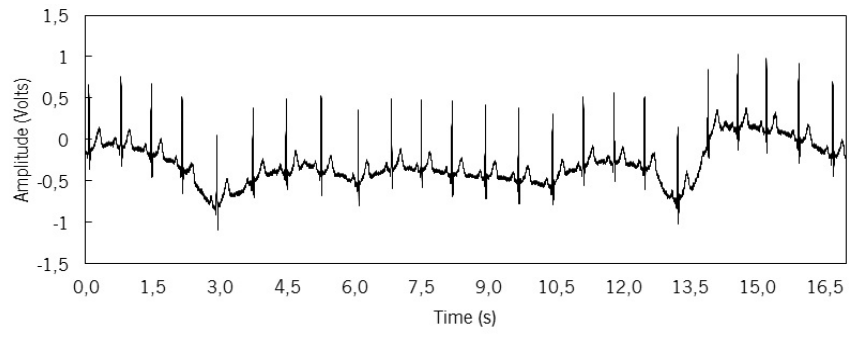

Fig. 14. Motion artifacts in ECG signal acquired with wet textile electrodes upon slow rotational movements of the body.

The electrical $50 \mathrm{~Hz}$ noise pick-up from the mains power was assessed acquiring ECG signals with a power cord, supplying power to a $2 \mathrm{~kW}$-heater, next to the body. The result is shown in Fig. 15.

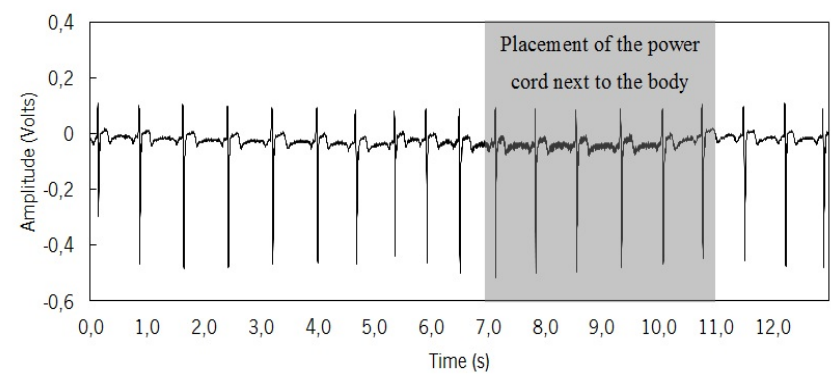

Fig. 15. $50 \mathrm{~Hz}$-noise picked up from a power cord.

Finally, the behavior of the system upon disconnection and reconnection of one of the electrodes was observed. The result is presented in Fig. 16.

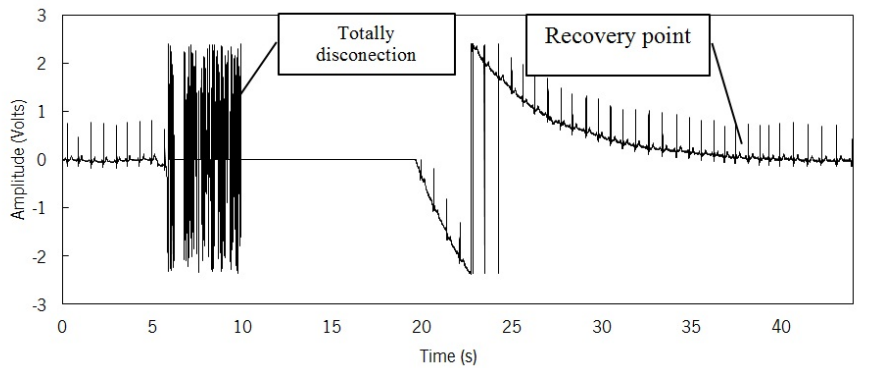

Fig. 16. ECG signal upon disconnection and reconnection of one electrode

\section{DISCUSSION}

As can be observed in the results presented in the previous section, the signal acquisition system, besides presenting very good CMRR and low noise levels, clearly depicts the waveforms of the ECG signal.

The textile electrodes provide a signal which is clear when they are wet, but in dry state, the signal quality tends to be worse.

Immunity to electrical noise has been found to be very good. Fig. 15 shows the marginal increase in $50 \mathrm{~Hz}$-noise even when a power cord is very close to the body. 
Motion artifacts, however, present a problem using the equipment. A further issue arises with electrode disconnection, a situation that can occur during use of the shirt with intensive physical activity, by a brief loss of contact between the e-textile electrode and the body. The ADS 1198 provides disconnection detection, switching off the output signal, but recovery upon reconnection takes about 15 to 20 seconds, which we consider quite long.

All of the signals presented are raw signals obtained directly from the acquisition system and can be significantly improved using standard filtering techniques. Valuable information can be obtained through feature extraction techniques previously used by the research team. These will be used for further evaluation of the system's performance.

The circuit has a very small footprint and can be supplied with voltage as low as $2.7 \mathrm{~V}$, allowing it to be placed near to the electrodes, and thus improving immunity to noise. Power consumption is very low, measured at only $10 \mathrm{~mA}$.

The possibility of changing the amplifier's gain through the SPI interface can be useful to apply the system to varying situations and measurements. The gain may be reduced, for instance, in situations in which the signal's baseline is unstable (heavy sports), or may be adapted to different measurements (EMG, EEG, ECG). It is also possible to easily expand the system to measurement of up to 8 differential channels.

\section{CONCLUSIONS AND FUTURE WORK}

In this work a small signal conditioning system was developed showing good performance in the acquisition of biosignals. The system operates on battery power and is ready to be integrated with a Bluetooth or Zigbee interface for maximum portability. Another possibility is its integration into a more complex system, served by a Body Area Network to which it may be connected through its SPI interface or again with a wireless solution. Its use with textile electrodes has been tested and will further be validated using standard equipment.

Future work should investigate the influence of the electrode positioning and ways of assuring compression of the electrodes against the body for higher signal quality and less sensitivity to motion. Force sensors will be used to assess the compression of the electrodes against the body, for different textile structures, materials and knitting parameters. Furthermore, it should be studied if the use of the integrated signal connections has any effect on signal quality, given that previous work [4] has shown more interesting results with textile electrodes.

The system will be equipped with an auxiliary circuit to temporarily reduce the time constant of the high-pass filter when electrode disconnection is detected, allowing faster recovery from electrode disconnection/reconnection, an important feature in some applications, such as biosignal monitoring in sports.

\section{REFERENCES}

[1] Catarino A., Carvalho H., Rocha A. M., Montagna G., Dias M. J., "Biosignal monitoring implemented in a swimsuit for athlete performance evaluation", Proceedings of AUTEX 2011 Conference, 8 10th June 2011,Mulhouse, France, pp 807-813

[2] Catarino, A.P., Rocha, A.M., Carvalho, H., Montagna, G. Dias, M.J., Covas H., Machado, R., Villas-Boas, J.P., Duarte J., Ribeiro S., Machado L., Borgonovo M., Fonseca P., Correia, M.V, "Integration of Biosignal Monitoring in Sports Clothing", Proceedings of the TRS2012The 41st Textile Research Symposium, September 12th to 14th, Guimaraes, Portugal

[3] Catarino, A., Carvalho. H., Barros, L., Dias, M.J., "Surface Electromyography Using Textile-Based Electrodes", The Fiber Society 2012 Fall Meeting and Technical Conference, Boston, USA, November 7-9, 2012

[4] Catarino, A, Carvalho, H, Dias, M.J., Pereira, T, Postolache, O., Girão P. S., "Continuous Health Monitoring Using E-Textile Integrated Biosensors", 2012 International Conference and Exposition on Electrical and Power Engineering (EPE 2012), 25-27 October, Iasi, Romania

[5] J. G. Webster, "Reducing motion artifacts and interference in biopotential recording," IEEE Transactions on Biomedical Engineering, vol. BME-31, no. 12, pp. 823 - 826, 1984.

[6] B. B. Winter and J. G. Webster, "Reduction of interference due to common mode voltage in biopotential amplifiers," IEEE Transactions on Biomedical Engineering, vol. BME-30, no. 1, pp. 58 - 61, 1983.

[7] J. C. Huhta and J. G. Webster, "60-hz interference in electrocardiography," IEEE Transactions on Biomedical Engineering, vol. BME-20, no. 2, pp. 91 - 101, March 1973.

[8] J. G. Webster, "Interference and motion artifact in biopotentials," Region Six Conference Record, IEEE, pp. 53 - 64, 1977.

[9] J. G. Webster, Medical Instrumentation, Application and Design, 3rd ed., J. G. Webster, Ed. John Wiley \& Sons, Inc, 1998.

[10] B. B. Winter and J. G. Webster, "Driven-right-leg circuit design", IEEE Transactions on Biomedical Engineering, vol. BME-30, no.1, 62 - 66, 1983.

[11] K. Mankodiya, Y. Ali Hassan, S. Vogt, H. Gehring, U.G. Hofmann, "Wearable ECG module for long-term recordings using a smartphone processor," In: Proc. 5th International Workshop on Ubiquitous Health and Wellness, UbiComp 2010, Copenhagen, Denmark, Sept. 26, 2010.

[12] R E.J.F.R. Aguirre, J. Moreno and M. F. Franco. "Design and construction of an electrocardiograph prototype with touch screen interface and embedded system with 16-bit resolution," XV Simposio de tratamiento de señales, imágenes y visión artificial - Stsiva 2012

[13] E. M. Spinelli, R. Pallàs-Areny, and M. A. Mayosky, "AC-coupled front-end for biopotential measurements," IEEE Transactions on Biomedical Engineering, vol. 50, no. 3, pp. 391 - 195, March 2003.

[14] A.C. Metting van Rijn, A. C., Peper, A., and C. A. Grimbergen, "High quality recording of bioelectric events. Part 1: Interference reduction, theory and practice," Medical and Biological Engineering and Computing, vol. 28, pp. 389-397, September 1990. 